\title{
Reasons of the transformed toward NOSQL Databases and its data models
}

\author{
Shaymaa Ahmed Razoqi \\ Department of Computer Science, College of Education for Pure Science, University of Mosul, Mosul, \\ Iraq
}

Email: shymaa.raazoqi@uomosul.edu.iq

(Received June 12, 2019; Accepted October 30, 2019; Available online June 01, 2020)

DOI: 10.33899/edusj.2020.165303, (C) 2020, College of Education for Pure Science, University of Mosul.

This is an open access article under the CC BY 4.0 license (http://creativecommons.org/licenses/by/4.0/).

\begin{abstract}
The relational database model is the main focus of the database for the previous time. It provides robust data storage and structure that supports transaction characteristics and data retrieval with structured query language- SQL. The emergence of web technologies and clustering technology in large servers called for the need to store unstructured data and move away from the pattern of tables and static fields and not topic to transaction conditions, especially in distributed systems. Instead, it used CAP theory. New data models are emergence including: Document Data model, Key-Value model, Column-Family model, and Graph model, And new programming languages that deal with these models.

An asset inventory system is a model of systems that are not topic to a fixed structure. Each location in the organization has fairly different assets and assets in the same location have different specifications that are difficult to organize in the form of tables and columns. For this reason using of NOSQL CouchDB database system was adopted and the use of MAP-REDUCE technology to organize data display and Cloudant Query in Mango-view method to retrieve data from the inventory database.
\end{abstract}

Keywords : Relational Database, NOSQL Databases, NOSQL Data Model, CouchDB DBMS

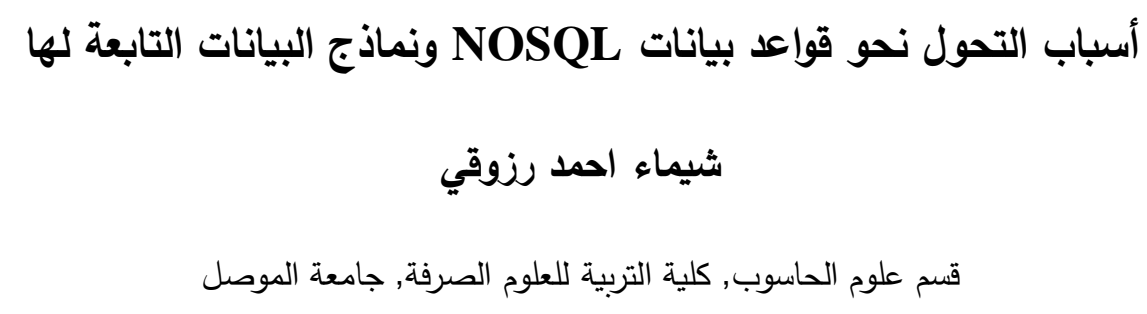

الخلاصة

يعتبر نموذج قواعد البيانات العلائقية هو المحور الأساس لقواعد البيانات طوال الفترة السابقة لكونه يوفر خزن وهيكلة بيانات قوية تدعم خصائص المعاملة وسهولة استرجاع البيانات بوجود لغة الاستعلام المهيكلة SQL.ظهور تقنيات الويب وتقنية. العناقيد في الخادمات الكبيرة دعت الحاجة الى خزن البيانات غير المهيكلة NOSQL 
الثابتة ولا تخضع لشروط المعاملة، خصوصا في النظم الموزعة، وبدلا من ذلك تستخدم نظرية الCAP وتبعا لذلك ظهر نماذج بيانات جديدة منها نموذج وثيقة البيانات ونموذج القيمة الرئيسة ونموذج عائلة الاعمدة ونموذج المخطط، وظهرت لغات برمجية جديدة تتعامل مع هذه النماذج.

نظام جرد الموجودات هو من نماذج الانظمة التي لا تخضع لهيكلة ثابتة فكل موقع في المؤسسة يضم موجودات مختلفة نوعا ما وكذلك الموجودات في نفس الموقع لها مواصفات مختلفة يصعب تتظيمها على شكل جداول من صفوف واعمدة ثابتة، لهذه الاسباب تم استخدام نظام قواعد بيانات NOSQL CouchDB واستخدام تقنية المقابلة والتخفيض لتنظيم عرض البيانات ولغة Cloudant Query بطريقة Mango-view لاسترجاع البيانات من قاعدة البيانات جرد الموجودات. الكلمات المفتاحية : قواعد البيانات العلائقية، قواعد بينات NOSQL، نماذج بيانات NOSQL، نظام ادارة قو اعد بيانات CouchDB

\section{1. مقدمة}

المتابع للتطورات التي حدثت في علوم وتقنيات الحاسوب يرى أن هناك تغيرات كبيرة ومتتوعة طرأت على مجالات اللغات البرمجية والهندة المعمارية، ولكن الثيء الوحيد الذي لم تطرأ عليه تغيرات كبيرة هي قواعد البيانات العلائقية (Relational Databases(RDB، وفيها البيانات المنظمة تدوم وتكون ذات خزن مستقر ، والذي يسهل الوصول إليها ومعالجتها بشكل جيد جدا من قبل العديد من التطبيقات البرمجية المتتوعة.

دفع تطور البرمجيات الحديثة وظهور كميات واسعة من البيانات الكبيرة لتغيرات أساسية في بناء القطع المادية، من ذلك مثلا بناء خادمات كبيرة تستخدم تقنية العناقيد) (Clusters) التي تتضمن بيانات غير منظمة وبكميات كبيرة جداً ومتتوعة، وتخضع لمعالجات وتحليلات مستمرة. الأمر الذي اوجب بالابتعاد عن قواعد البيانات العلائقية وشروط المعاملات(Transactions) ولغة الاستعلام المهيكلة (Structure Query language -SQL)، ولكن هذا لا يعني أن قواعد البيانات العلائقية سوف تتوقف. فمن المتوقع أن تبقى مستعملة لعقود قادمة، وستبقى هي المسيطر الرئيس لقواعد البيانات المنظمة على الرغم من ظهور تقنيات قواعد بيانات NOSQL واستخدام نظرية الCAP وتنوع نماذج البيانات غير المهيكلة، وتبعا لهذا ظهرت لغات برمجة جديدة وبرمجيات لإدارة قواعد البيانات مثل CouchDB و MongoDB و Cassandra.

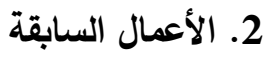

اقترح Vera [1] وآخرون طريقة لنمذجة بيانات NOSQL والغرض منها هو إعطاء رؤيا واضحة للبيانات بالإضافة إلى التأكيد على أهمية أسلوب نمذجة البيانات وحالة الدراسة التي تم اعتمادها كانت استخدام بيانات جينية. هig- هalehnia [2] لإيجاد طريقة لتحويل قواعد البيانات العلائقية إلى بيئة البيانات الكبيرة . Data البيانات الكبيرة بالإضافة إلى عمل مقارنة بين البيانات المهيكلة وشبه المهيكلة وغير المهيكلة . 
قدم Chandra [3 دراسة تحليلية للخصائص الأساسية لقواعد بيانات NOSQL مع التركيز على برمجياتها الواسعة النطاق واستخدام تقنيات مختلفة لتحقيق اتساق البيانات، وأيضا تقنيات توفر البيانات مقارنة مع خصائص ACID لقواعد البيانات

العلائقية.

قدم Meijer [4] واخرون مقارنة بين ثلاثة أنواع من قواعد البيانات وطريقة أدائهم النسبي مع الأخذ بنظر الاعتبار لطريقة خزن البيانات، وكذلك مقارنة تنفيذ عمل هذه القواعد على خادم فيزيائي وعملهم على آلة افتراضية بالنسبة لعمليات القراءة المفردة والكتابة المفردة وكذلك عمليات القراءة والكتابة المتعددة. كثف Varga [5] وآخرون في الورقة التي قدموها طريقة عرض تصوري تقليدي لقواعد بيانات الوثيقة باستخدام تحليل المفاهيم الرسمي ومدى قدرته على إعطاء صورة مرئية كاملة لتحليل البيانات واسعة النطاق.

3. قواعد البيانات العلائقية

قواعد البيانات العلائقية أصبحت جزءاً ضمنياً في أغلب التطبيقات البرمجية ولا يمكن الاستغناء عنه حسب تصور أغلب المبرمجين، وذلك لسهولة استعمالها وتعاملها مع البيانات بأسلوب منظم وخزن ثابت فهي توفر استرجاع بيانات ثابت، وهي في نفس الوقت تسمح للعديد من المستخدمين بالوصول إلى نفس البيانات بثكل متزامن مع إمكانية تعديل تلك البيانات[2]. إن أغلب التطبيقات الحديثة لقواعد البيانات العلائقية تكتب من قبل فرق مختلفة من المبرمجين، وتستخدم هذه التطبيقات نفس البيانات،لذا فالتغير الذي يحدثه أي تطبيق يجب أن يكون مرئياً لبقية التطبيقات.والطريقة المستخدمة لذلك هي تكامل قاعدة البيانات المشتركة وهي أكثر من تطبيق يخزن البيانات نفسها، والتي تعامل بها تعدد المستخدمين في تطبيق واحد [6]. نجحت قواعد البيانات العلائقية بثكل كبير كونها تستخدم نماذج قياسية رئيسة مع اختلافات بسيطة جداً، ولكن الميكانيكية الأساسية هي نفسها وكذلك طرق التعامل مع المناقلات. ومنذ بداية قواعد البيانات العلائقية كانت هناك مشكلة الاختلاف مع تركيب الذاكرة، ففي النموذج العلائقي كل شيء ينظم في جداول (القيم فيها بسيطة غير مركبة) وعلاقات، وتعتمد على الجبر العلائقي، وهذا يعطي مرونة وبساطة في المعالجة ولكنه ينتج محددات وتعقيدات بثكل خاص عند التعامل مع التركيب الهيكلي للذاكرة حيث من الممكن يأخذ هيكل أغنى[7]. العامل الرئيس لبقاء قواعد البيانات العلائقية عبر الزمن هو قوانين لغة الاستعلام المهيكلة SQL والآلية تكامل قاعدة البيانات مع التطبيقات المختلفة. وقاعدة البيانات التي يتم بناؤها لأداء مجموعة أغراض تخزن كميات كبيرة من البيانات في مكان واحد مشترك تصله مجموعة من التطبيقات البرمجية التي يتم تصميمها للتعامل مع قاعدة البيانات هذه. طريقة قاعدة البيانات المشتركة مفيدة من حيث تحسين الاتصال وتوفير موقع الخزن، لكنها تأتي مع سلبيات منها عدم قدرة احد التطبيقات لتغيير تتسيق البيانات -إذا احتاج لذلك- إلا بما يتناسب مع بقية التطبيقات التي تستعمل قاعدة البيانات ولا يتعارض معها.

مع بداية الألفية الثانية بدأ استخدام تطبيقات الويب وبروتوكولات HTTP لنقل البيانات وبدأت هذه التطبيقات الجديدة تمثل تحدياً جديداً لاستعمال SQL مع قواعد البيانات المشتركة، ونتج عن هذا الانتقال مرونة أكثر لهياكل البيانات التي بدأت

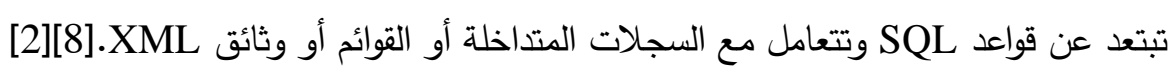


4. - M ظهور تقنية العناقيد

مع ظهور الألفية الجديدة بدأت الثبكات الحاسوبية بالانتشار وتوسعت خدمات الويب وتتوعت وظهرت برمجيات التواصل الاجتماعي، التي أدت إلى ظهور مجموعات كبيرة من البيانات، ومع النمو في البيانات جاء أيضا تزايد ملحوظ في عدد المستخدمين والزوار لمواقع الويب، ونتيجة لزيادة حجم البيانات والطلبات زادت الحاجة إلى استعمال مصادر الحاسبات بشكل أكبر ، ولمعالجة هذا النوع من الزيادة ظهر خياران:

الاختيار الأول هو استخدام آلات حاسبة كبيرة ومعالجات أكثر قوة وأقراص خزن وذاكرة أوسع، ولكن هذا الحل يحتاج كلفة أكبر • والاختيار الثاني هو استخدام عناقيد من آلات حاسبة صغيرة قليلة الكلفة، وتكون أكثر مرونة، وتكون حالات الفشل فردية، إذ ان بقية الأجهزة في العنقود يمكن أن تصمم لكي تستمر بالعمل حتى مع فثل أحد الأجهزة، وهذا يعطي مستوى عالياً من الثقة، حيث بدأ الاتجاه العناقيد وهذا أدى لظهور مشكلة جديدة وهي ان قواعد البيانات العلائقية ليست متهيئة للعمل في بيئة العناقيد [9].

\section{NOSQL 1-1 ظهور قواعد بيانات}

ظهرت هذه التسمية أول مرة في التسعينات كاسم لقواعد بيانات مفتوحة المصدر تحت قيادة Carlo-Strozzi [9]. قواعد البيانات هذه تخزن جداولها كملفات اسكيASCII، وكل سجل يمثل سطراً من البيانات بثكل حقول تفصلها فراغات. وجاء الاسم من حقيقة ان قاعدة البيانات لا تستخدم لغة الاستعلام المهيكلةSQL. وبدلاً من ذلك عولجت قاعدة البيانات من خلال المستتدات المغلقة والمدمجة مع خطوط يونكس العادية[2].

مع بداية الألفية الجديدة الاسم NOSQL بدأ يعبر عن شيء مختلف نوعاً ما عن السابق، إذ ان هذه التقنية الجديدة ظهرت على يد Johan Oskarsson . والتعبير انتشر بشكل كبير لكن لم يكن هناك تعريف واضح، فهو ناتج عن البحث عن قواعد بيانات غير علائقية موزعة مفتوحة المصدر ، لذا فكل ما هو معروف عنها هو مجموعة خصائص مشتركة لقواعد بيانات التي تميل إلى اسمNOSQL [3]، واهم تلك الخصائص:

• ان قواعد بيانات NOSQL لا تستخدم لغة الاستعلام المهيكلةSQL. وهذه تعتبر النقطة الأكثر وضوحاً على الرغم من أن البعض منها تستخدم لغة استعلام خاصة بها مشابهة للغة الاستعلام المهيكلة لكنها أسهل في التعلم مثل لغة استعلام

Cloudant Query كساندرا CQL ولغة والخاصية الثانية لقواعد البيانات هذه أنها قواعد مفتوحة المصدر . • خاصية أخرى تثترك بها معظم قواعد NOSQL تقود إلى الحاجة للتنفيذ على العناقيد، وهذا له تأثير على نماذج البيانات بالإضافة إلى تتاست البيانات. في حين تستخدم قواعد البيانات العلائقية خصائص المعاملة ACID (الوحدة Atomicity، والاتساق Consistency، والعزل Isolation، والمتانة Durability) لمعالجة تتاسق البيانات عبر تكامل قواعد البيانات، وهذا يتعارض مع بيئة العناقيد، 
فإن قواعد بيانات NOSQL لا تعطي أهمية لخصائص المعاملة وفي بعض الحالات تتجاهلها تماماً خصوصاً في النظم الموزعة، والتي يكون فيها غالباً من غير الممكن ضمان الالتزام بهذه الخصائص[10]. قواعد بيانات NOSQL تقدم مدى من الاختيارات

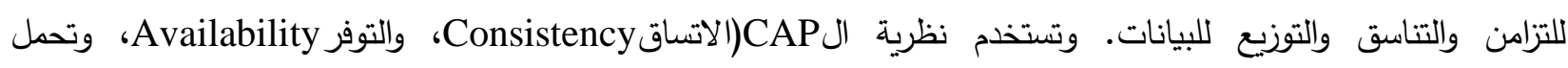
التقسيم Partition tolerance)، والتي قدمت سنة 2000 من قبل Eric Brewer [12]، وأُثبتت فيما بعد من قبل Seth، Nancy Lynch و Gilbert زمن معين ولكن ليس الثلاثة معاً[11]. وعملية الاختيار يمكن أن تتم كالتالي: • التوفر يمكن أن يتم تجاهله ويفضل عليه كل من الاتساق وتحمل التقسيم. الاتساق مع التوفر يكون مفضلاً إذا كان النظام فيه تقسيماً قليلاً أو غير مقسم.

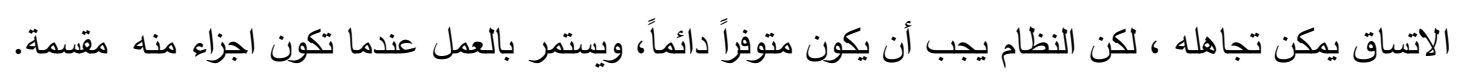

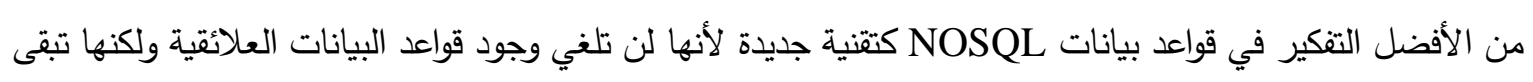
المساهم الأكبر لأنواع قواعد البيانات المستخدمة في الوقت الحالي فهي توفر دعماً وأمناً واستقراراً لأغلب المشاريع. ومع وجود تلإد

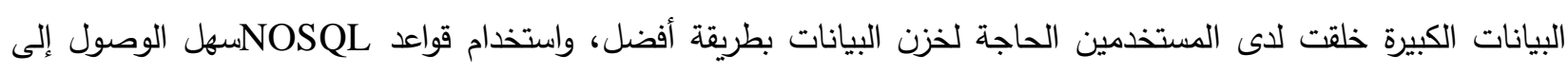
البيانات، وساعد في زيادة معدل إنتاج المعرفة. قواعد بيانات NOSQL تعمل بدون أي هيكلية، وتسمح بإضافة حقول جديدة إلى الجداول بدون تغير في الهيكل الأصلي أو التطبيقات البرمجية وهذا، مفيد عند التعامل مع بيانات ليس لها شكل منظم رسمي وحقول حسب الطلب[9].

NOSQL 4-2 نماذج البيانات في قواعد بيانات

تسمى طريقة تصميم وتعريف ومعالجة البيانات بصورة ما بنموذج البيانات وكيفية وصف البيانات، وكيف سيتم التفاعل مع البيانات في قاعدة البيانات، وهذا يتم بمعزل عن نماذج الخزن التي تصف كيف يتم خزن قاعدة البيانات، وكيف تعالج البيانات

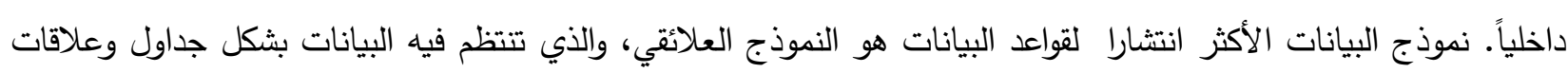
بينها، وكل جدول يتكون من صفوف وأعمدة تتقاطع مكونة خلايا تحمل كل منها قيمة وحيدة . النموذج العلائتي يأخذ المعلومات المراد تخزينها ويقسمها إلى جداول مكونة من صفوف والصفوف هي هياكل بيانات محددة تأخذ بيانات بسيطة، ولا يمكن مراكمة صف فوق آخر للحصول على سجلات متراكمة أو متداخلة ولا يتم وضع مجموعة

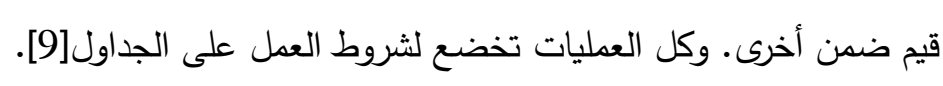

المجموعات الموجهة كنموذج بيانات جديد تأخذ طريقة مختلفة، فهي تعمل على بيانات تكون بشكل هياكل أكثر تعقيداً من الجداول تسمى وحدة المجموعة، والتي تعرف على أنها وحدة بيانات تستخدم للمعالجة والتتاسق والتكامل، وتتكون من عناصر مترابطة تعامل كوحدة مستقلة واستخدام هذا النوع من وحدات البيانات يتتاسب مع أنواع قواعد NOSQL.المجموعات الموجهة هي نماذج بثكل مجموعات موجهة قوية وكل منها له رمز تعريفي يستخدم لاسترجاع البيانات، وهنا يكون من الممكن خزن أي أنواع البيانات في المجموعة الواحدة، قاعدة البيانات قد تعرض حجماً عاماً محدداً للمجموعة الواحدة[13]. 
في قواعد بيانات NOSQL يُرى بشكل واضح الابتعاد عن النموذج العلائقي والانتقال إلى نماذج مختلفة أخرى يمكن وصفها في أربع نماذج واسعة للاستخدام في هذه البيئة، والذي يربط عناصر البيانات المختلفة عند التخطيط للنموذج العلائقي هي المفاتيح في حين لا يوجد شيء مماثل لتمييز العلاقات التي تربط المجموعات مع بعضها في قواعد بيانات المجموعات

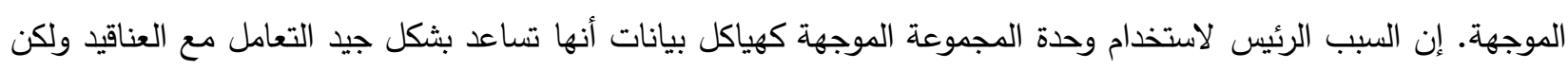
يتم السعي دائما إلى تقليل عدد العقد للاستعلام الواحد عندما تجمع البيانات من العناقيد. لذا هناك حاجة دائما إلى دراسة أنواع الاستعلامات ووضع البيانات التي سوف تطلب مع بعضها في نفس العقدة أو المجموعة[10].

استخدام وحدة المجموعة لها نتائج مهمة للمعاملات. وكما هو معروف قواعد البيانات العلائقية تسمح بمعالجة مجموعة من السجلات (صفوف) من أي جدول في معاملة واحدة، وهذا يخضع لشروط المناقلة ولكن قواعد بيانات لات لدمعم هذه الثروط، وهذا معناه التضحية بالتتاسق كشرط لتنظيم البيانات وبذلك فهي تكون أسرع في معالجة المجموعات المتعددة، وبدلاً من

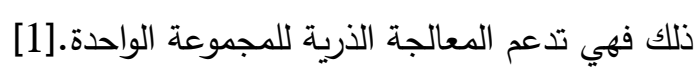

\section{Document Data Model 4-2-4 نموذج وثيقة البيانات}

يتم تخزين البيانات في هذا النوع على شكل ملفات لا تعتمد على تصميم ثابت، وكل ملف يمكن ان يحتوي على تصميم مختلف وغير منظم. قاعدة بيانات الوثيقة الموجهة كما يدل الاسم نموذج خزن البيانات المطلوبة على شكل وثيقة، وهي تفرض قيوداً على كل مايمكن خزنه بالمقابل هناك مرونة أكثر في الوصول للبيانات. ضمن نموذج بيانات الوثيقة الموجهة في قواعد بيانات NOSQL تكون البيانات غير منظمة ولا تتبع صيغة معيارية ثابتة أي تكون شبه مهيكلة[14]. مثلا إذا كان لدينا مكتبة كتب تخزن معلومات الكتب بشكل وثائق تسمى Book وضمن هذه الوثيقة يتم خزن اسم الكتاب وأسماء المؤلفين وتاريخ النشر واسم دار النشر والطبعة وغيرها من معلومات الكتاب، كذلك يمكن خزن مؤشرات لوثائق أخرى، وهذه المؤشرات تكون بمكانة المفاتيح الأجنبية لقواعد البيانات العلائقية، يوضح (الثكل1) نموذج بيانات الوثيقة الموجهة.كل من برنامج MongoDB وبرنامج

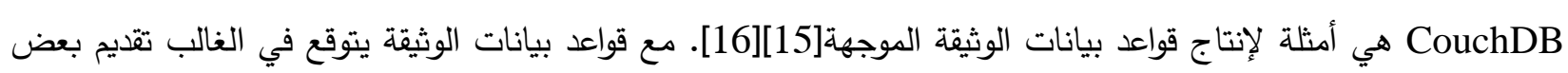
النماذج للاستفسار بالاعتماد على الهيكل الداخلي للوثيقة[17].

Book Title: Business Intelligence and Analytics: Systems for Decision Support

By Ramesh Sharda, Dursun Delen, Efraim Turban

Publication Date: 2015

Edition: $10^{\text {th }}$

Publisher: Pearson

Publisher Location: Upper Saddle River, NJ:.

ISBN-13: 978-0-13-305090-5

$$
\text { شكل(1) نموذج بيانات الوثيقة }
$$


Key-Value Modelaئيسة

تخزن البيانات في نموذج القيمة الرئيسية بشكل أزواج متتاظرة من الأعمدة (عمودين متتاظرين) ، يضم العمود الأول قيمة المفتاح، ويضم العمود الثاني القيمة الفعلية للبيانات. القيمة الفعلية للبيانات يمكن أن تكون نصاً بسيط، أو أنواع بيانات مركبة معقدة. في طريقة القيمة الرئيسة للخزن يمكن فقط الوصول إلى المجموعة بالبحث عن مفتاحها.مع قواعد بيانات القيمة الرئيسة يتوقع في الغالب البحث في التجمعات باستخدام المفتاح. وتكمن قوة قواعد بيانات القيمة الرئيسة في سرعة القراءة، إذ يتم استخدامها في عملية التخزين المؤقت للبيانات[16][10].

من الأمثلة لقواعد بيانات NOSQL التي تستخدم نموذج بيانات القيمة الرئيسة هي Project Voldemort, Cache and Dynamo. في المثال السابق لتمثيل بيانات الكتاب باستخدام نموذج بيانات القيمة الرئيسة يمكن توضيحها بـ(الثكل2) [17].

\begin{tabular}{||l|l||}
\hline Key & Value \\
\hline Book Title & $\begin{array}{l}\text { Business Intelligence and } \\
\text { Analytics: Systems for De- } \\
\text { cision Support }\end{array}$ \\
\hline Author (set) & Ramesh Sharda \\
\hline & Dursun Delen \\
\hline & Efraim Turban \\
\hline Publication Date & 2015 \\
\hline Edition & $10^{\text {th }}$ \\
\hline Publisher & Pearson \\
\hline$\ldots$ & $\ldots$ \\
\hline
\end{tabular}

شكل(2) نموذج بيانات القيمة الرئيسية

Column-Family Stores

تستخدم جوجل قواعد بيانات NOSQL يسمى جداول جوجل الكبيرة واسمه استخلص من الهياكل المجدولة، والتي تكون بشكل أعمدة متناثرة وليس لها اسكيما، ولا يمكن التفكير في هذا التركيب كجدول وبالأحرى هو خريطة بمستويين. أي جداول الأعمدة فيها من نوع جدول، ومن اشهر الامثلة على هذه النظم قواعد البيانات التالية مثل كاسندرا[18][16].معظم قواعد البيانات العلائقية يكون فيها الخزن على شكل سطر كوحدة خزن، والذي يساعد في تحسين الأداء. في معظم الاستعلامات عن البيانات يتم جلب البيانات من أكثر من جدول (أي عدة اعمدة من عدة جداول في المرة الواحدة)، لهذا الغرض من الأفضل خزن مجموعة أعمدة لكل السطور في وحدة خزن واحدة. لذا تسمى هذه الطريقة مخازن الأعمدة وطريقة الجداول الكبيرة جاءت من هذه الفكرة، وهي خزن مجموعة أعمدة مع بعضها كعائلة واحدة.

نموذج بيانات عائلة العمود له صيغة خزن بيانات مشابهة إلى حد ما لقواعد البيانات العلائقية ، بالرغم من ان قواعد بيانات العلائقية تميل إلى التعامل مع أنواع بيانات بسيطة وذات هيكل منظم ومعروف مسبقا، قواعد بيانات NOSQL عائلة 
العمود توفر مرونة أكثر بكثير، فهي تدعم أنواع بيانات مركبة ومعقدة، وغير مهيكلة[15]. يبين (الثكل3) نموذج عائلة العمود، وكل من (المؤلف وتاريخ النشر والطبعة والناشر ) قد تم تضمينها في نوع بيانات مركب يدعى تفاصيل الكتاب( book

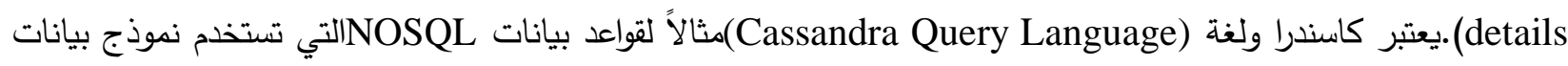
عائلة عمود[17][19].

\begin{tabular}{||l||}
\hline \multicolumn{2}{|l||}{ Business Intelligence and Analytics: Systems for Decision Support } \\
\hline Book Details (includes authors, year, edition, publisher, etc.) \\
\hline Ramesh Sharda \\
\hline Dursun Delen \\
\hline Efraim Turban \\
\hline 2015 \\
\hline $10^{\text {th }}$ \\
\hline Pearson \\
\hline \hline
\end{tabular}

الثكل(3) نموذج عائلة العمود

كما في مخازن القيمة الرئيسة المفتاح الأول يصف معرف السطر ـ والفرق مع عائلة الأعمدة في ان مجموعة الأسطر نفسها تصف الخريطة لتفاصيل القيم، أما قيم المستوى الثاني فهي تشير إلى الأعمدة ـ طريقة قواعد بيانات عائلة الأعمدة تنظم الأعمدة في عوائل كل عمود هو جزء من عائلة معينة. ويكون وحدة وصول، لذلك فالقيم المخزونة في الأعمدة المتثابهة في نفس العائلة سوف يتم الوصول لها مع بعضها.

\section{GraphModel}

يتم خزن البيانات في هذا النموذج على شكل عقد وعلاقات تربط العقد مع بعضها البعض. نموذج بيانات المخطط البياني يدعم خزن البيانات التي لها عدد غير معروف من الارتباطات في شبكة الانترنت. مثل بيانات الخرائط و خطوط النقل، وعلاقات الأوساط الاجتماعية[15][16] على سبيل المثال عمل رسم بياني لإيجاد المسافة الأقصر بين المدن يكون صعبا جدا باستخدام نموذج قاعدة البيانات العلائقية التقليدية، في حين قواعد بيانات NOSQLالدخطط البياني يمكن أن تسهل هذا النوع من المعالجة. من أمثلة قواعد البيانات التي تستخدم نموذج المخطط البياني هي Neo4j ونظام Virtuoso [110.

Schema less Databases قواعد بيانات بدون مخطط.

في قواعد البيانات العلائقية يستخدم مخطط Schema وتستخدم لعدد من التطبيقات باستخدام نفس قاعدة البيانات بدون تغير في التركيب وبسهولة، ولان هناك معرفة مسبقة بتظيم الجداول والأعمدة والعلاقات، أما في قواعد بيانات لا يوجد مخطط، وهنا سوف يتم الاعتماد على التطبيقات في الوصول السليم للبيانات، أي يتم عمل زحفاً للمخطط من قاعدة بيانات نحو 
التطبيقات البرمجية وطريقة الوصول ، أي الاعتماد على التطبيقات في الحصول على خزن جيد واسترجاع جيد، فهنا لا توجد طريقة محددة مسبقة تعتمد على التطبيق، وتظهر المشكلة في حالة وجود أكثر من تطبيق يريد الوصول إلى نفس البيانات[1]. إحدى طرق معالجة المشكلة هذه استخدام طريقة كبسلة وتغليف قاعدة البيانات في احد التطبيقات واستخدامها من قبل تطبيقات أخرى عبر هذا التطبيق باستخدام خدمات الويب[11]. 6.استخدام المشاهدViewsفي قواعد البيانات

تسمح قواعد البيانات العلائقية بالوصول إلى البيانات بطرق مختلفة وتوفر تقنيات تسمح بالبحث بطرق مختلفة مثل استخدام المشاهد. والمشهد يثبه الجدول أو العلاقة، ولكن يتم تعريفه برمجياً والمشاهد تخفي المكان الحقيقي لخزن البيانات، وكذلك طريقة هيكلة قاعدة البيانات، ولكن بعض المشاهد مكلف برمجيا [15].وبالرغم من أن NOSQLليس فيها مشاهد كما في ولكن فيها إمكانية تكوين استفسارات محسوبة مسبقاً ومخزونة ويمكن استخدام نفس التعبير ظاهرة المشاهد. عادة قواعد

[14].Map-Reduce تكون ظاهرة المشاهد باستخدام حساباتNOSQL بيانات

هناك نظريتان لبناء ظاهرة المشهد في قواعد بيانات NOSQL، الأولى النظرة المتلهفةEager حيث يتجدد المشهد في نفس اللحظة لتجدد البيانات الأصلية له. وهذه الطريقة جيدة عندما يكون معدل القراءات أكثر من معدل الكتابة للبيانات في المجموعة أو المشهد، والمطلوب دائما أن يكون المشهد متجدداً قدر الإمكان[16].النظرة الثانية قاعدة بيانات التطبيقApplication database هي نظرة الثيء الثمين، هنا الأمر أكثر سهولة لضمان أي تغيير أو تعديل للبيانات الأصلية سوف يعدل المشهد مجداً. ولتقليل زيادة الحمل عند كل تحديث يمكن عمل أو تتفيذ دفعة أعمال لتحديث عدة مشاهد عند مرور فترة زمنية معينة، وهذا يتطلب معرفة متطلبات العمل بثكل دقيق وكيفية وعدد مرات الوصول للبيانات، وكيف يتم تكوين المشاهد. ويمكن تكوين المشهد خارج حدود قاعدة البيانات باستخدام تطبيق برمجي وخزن المشهد بعدها في قواعد البيانات.ومعظم قواعد البيانات تدعم تكوين المشاهد بنفسها وتجهز عمليات حسابية لذلك عند الحاجة وحسب الطلب والمتغيرات المحددة.

Map-Reduce 1-6 تقنية المقابلة والتخفيض

Map- هي معالجة تقنية أو برنامج يستعمل كنموذج في الحسابات الموزعة، تتضمن خوارزمية المقابلة والتخفيض Reduce البيانات وتحويلها إلى مجموعة أخرى، إذ أن عناصر البيانات الفردية تحول إلى أزواج من البيانات Tuples مكونة من (مفتاح

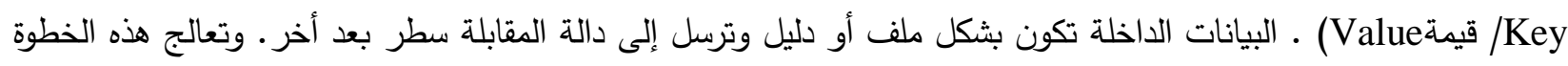
البيانات وتتتج قطع صغيرة من البيانات المسماة Chuncks.

الخطوة الثانية هي عملية التخفيض، وهي تأخذ ناتج عملية المقابلة كإدخال وتتضمن مرحلة الخلط والتخفيض للبيانات. تقوم هذه العملية بدمج أزواج البيانات القادمة من عملية المقابلة إلى مجموعة اصغر من الأزواج الجديدة. كما يدل الاسم فان مهمة التخفيض لا تبدأ إلا بعد انتهاء مهمة المقابلة، وان النظام هو المسؤول عن إرسال مهمات المقابلة والتخفيض إلى الخادم 
المناسب في العنقود. لذلك تكون هذه التقنية مناسبة جداً للعمل في بيئة العناقيد إذ يمكن بسهولة حساب أي معالجة موزعة للبيانات عبر عقد متعددة تتألف من مئات أو آلاف المعالجات في العنقود الواحد وهذا أدى إلى انجذاب المبرمجين لاستعمال نموذج المقابلة والتخفيض[20].

Data Consistency تناست البيانات

واحد من التغييرات الكبيرة من قواعد البيانات العلائقية إلى قواعد بيانات NOSQL هي كيفية التفكير تجاه التتاسق أو الاتساق. قواعد البيانات العلائقية تحاول بقوة الحفاظ على التتاسق وتجنب التضارب، وهناك أنواع مختلفة للتناسق منها ما يتعلق بعمليات التحديث أو القراءة] [21].

\section{Update Consistency 1-7 تناسق التحديث}

يفقد التتاسق عادة عندما يحصل تضارب في الكتابة Write-Write Conflictويتم ذلك عندما يأتي شخص بالكتابة على مقطع بيانات، ويأتي شخص ثاني بالكتابة على نفس مقطع البيانات، عندها سوف يلاحظ ان التحديث الثاني سيتم إلغاؤه

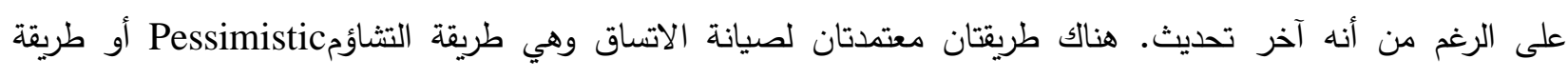
التفاؤلOptimistic تجاه التقاء الكتابة.طريقة النظرة المتثائمة تعمل بمنع النزاع من الحصول نهائيا، أما طريقة النظرة المتفائلة فهي تسمح بحدوث النزاعات وبعد اكتثافها تعمل على طرق لفك النزاع.[3]

في نزاعات تحديث النظرة المتثائمة الأكثر شيوعاً هي قفل الكتابة، ولأجل تحديث أي بيانات يجب امتلاك قفل الكتابة، وعندها النظام يضمن أن نقطة طرفية واحدة فقط هي التي تملك حق الكتابة في وقت معين. بينما النظرة المتفائلة هي الثائعة، وتتم عن طريق التحديث الثرطي، فأي نقطة طرفية تريد عمل تحديث يجب ان تفحص أولا القيمة قبل التحديث لرؤية التغيير على البيانات منذ آخر قراءة .كلتا النظريتين السابقتين تعتمد تسلسلات ثابتة للتحديث وهذا مع خادم يمكن معالجته الطرف الأول فالثاني وهكذا. لكن مع وجود أكثر من خادم في النظام والبيانات تستخدم طريقة الند للند لتكرار البيانات بين عدة نقاط عندها لو كان هنالك طرفيتان تعملان على نفس البيانات كل منها على خادم مختلف تكون النتيجة تغير القيم بطريقة مختلفة في كل خادم.[4]

\section{Read Consistency 2-7 تضارب القراءة}

عندما يكون هناك شخص يقوم بعملية كتابة وأخر يقوم بعملية قراءة لنفس البيانات في ان واحد فتكون هناك قراءة متتاقضةnconsistent readأو ما يسمى تضارب قراءة كتابةRead-Write Conflictويثار إلى هذا النوع من تتاسق البيانات المنطقيLogical Consistency

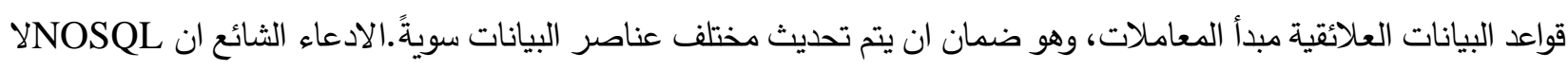
تدعم المعاملة وهذا لا يمكن ان يكون متوافق. إن هذا الادعاء خاطئ لأنه لا ييرر الكثير من التفاصيل[5].وقواعد بيانات المخطط البياني تميل إلى دعم المعاملة في حين تدعم قواعد بيانات المجموعة الموجهة التحديث الذري لكن فقط مع المجموعة الواحدة. 
أي هنالك تتاسق منطقي في المجموعة الواحدة وليس بين المجموعات. وبالطبع لا يمكن وضع جميع البيانات في مجموعة واحدة، لذا فان أي تحديث يؤثر على عدة مجموعات يترك المجال مفتوحاً لحدوث قراءات متضاربة عندما يقوم أكثر من زبون بالوصول إلى نفس البيانات[7] ـ يدعى طول الوقت (المدة الزمنية) لحدوث التضارب الحالي يدعى نافذة التضارب Inconsistency

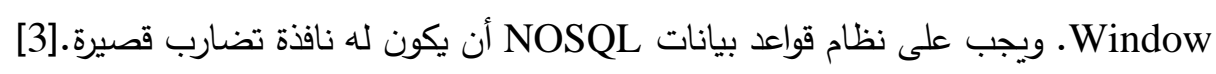

3-7 تناسق التكرار

وهو ضمان أن كل النسخ يصلها نفس التحديث لنفس البيانات في وقت واحد. مثلا اذا كان هناك ثلاث نقاط كل منها في منطقة جغرافية مختلفة، فعندما تريد اثنان من النقاط اجراء قراءة والنقطة الثالثة تنفذ تحديث في نفس الوقت سيتم ملاحظة أن النقطة الاقرب جغرافيا سوف ترى تحديثاً اسرع من النقطة الأبعد، لذلك يحصل تضارب تكرار . وفي النهاية تلقائيا كل التحديثات ستنتشر بالكامل على كافة النقاط. لذا تدعى هذه الحالة التوافق النهائي، أي مهما كان هناك من تضارب تكرار فإنه وبعد انتهاء

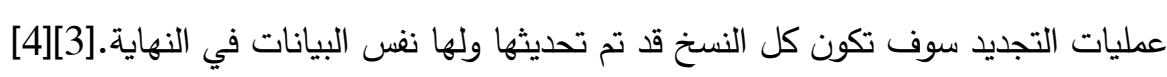

\section{8.نظام إدارة قاعدة البيانات NOSQL(CouchDB)}

هو نظام إدارة قاعدة بيانات NOSQL تختص بشكل كامل بخزن البيانات باستخدام نموذج وثائق JSON، وهو لا يتطلب هيكلاً خاصاً لجميع الوثائق[22]. نظام CouchDB يمكن أن يضم أكثر من قاعدة بيانات واحدة في نفس الخادم، وهو يدعم التوزيع لقاعدة البيانات باستخدام تقنية العنقدة وعمل التكرار Replication ويوفر نظام إدارة التضاربات ( اكتثاف ومعالجة النزاعات في قاعدة البيانات بصورة تلقائية)[5]. تعد الوثيقة الوحدة الرئيسة لقاعدة البيانات، وكل وثيقة لها رمز مميز (ID) وحيد غير قابل للتكرار في قاعدة البيانات. وتتكون الوثيقة الواحدة من عدد غير محدد من الحقول والمؤشرات. الحقول في الوثيقة ليس لها شروط ثابتة أو أسماء محددة وتضم أنواعاً مختلفة من البيانات، ولا يوجد حد معين لحجم النص أو عدد عناصر المصفوفة، ويمكن تكرار اسم الحقل مع نوع بيانات مختلف[23].

يوفر نظام CouchDB طريقة جديدة لعرض بيانات قاعدة البيانات بشكل تفاعلي غير مشابهة لطريقة البرمجة المهيكلةSQL، إذ إن طبيعة بيانات الوثائق شبه مهيكلة وتختلف عن طبيعة بيانات الجداول المهيكلة[1][5]. يمكن الاستعلام عن البيانات المخزونة في قاعدة البيانات CouchDB باستخدام جمل بناء استعلامات يتم تتفيذها عبر لغة جافا سكربت وضمن نظام عمل طريقة Map-Reduce إذ تكون الوثيقة ادخالاً وتجري عليها المقارنات المطلوبة على حقول الوثيقة، وتحدد فيما إذا كانت هذه الوثيقة جزء من النتيجة أم لا. وتبنى الاستعلامات (المشاهد view) بشكل ديناميكي ولا تؤثر على الوثائق الأصلية، وكذلك فإن المشاهد التي يتم بناؤها في إحدى نسخ قاعدة البيانات تكون خاصة بها ولا يتم مزامنتها مع باقي النسخ.يوفر نظام CouchDB العديد من البرمجيات التي تسهل التعامل مع إدارة قواعد البيانات منها Faoxton وهو واجهة تطبيق سهلة الاستخدام ويوفر وصولاً كاملاً لكل عناصر ووظائف CouchDB 
Inventory DBS تكوين قاعدة البيانات جرد الموجودات.9

نظام جرد الموجودات هو نظام يعمل على إحصاء الموجودات الثابتة والمتحركة في أقسام المؤسسة حسب موقع تواجدها. وتتم عملية الجرد بتسجيل كافة الموجودات مع تفاصيلها المتواجدة في أحد أجزاء بناياتها ، ويقوم بهذه المهمة مجموعة من الأشخاص يثكلون أعضاء لجنة الجرد. تتم عملية الجرد عادة عند نهاية كل سنة أو عند ورود تغيرات أو تحديثات على موجودات المؤسسة. تتم عملية الجرد بتسجيل كافة المواد العينية على استمارات خاصة معدة مسبقا من قبل لجنة الجرد.

\section{1-9}

من أجل تحويل قاعدة بيانات الجرد إلى نظام حاسوبي باستخدام النموذج العلائقي يتم جمع وتحليل البيانات وتنظيمها بثكل مهيكل (جداول وعلاقات) ولأجل ذلك يتم عمل جدول بالمواد(الموجودات) وأماكن تواجدها ومواصفات كل منها. ومن الملاحظ أن الموجودات تختلف عن بعضها البعض في عدد ونوع المواصفات، وبذلك فمن الصعوبة تكوين جدول بالموجودات ومواصفاتها مثلا جهاز الطابعة يختلف بعدد مواصفاته عن الكرسي ويختلف عن جهاز التكييف. ولأجل حل هذه المشكلة يمكن تحديد اكبر عدد مسموح للصفات ليكن مثلا 5، هذه الطريقة سوف تسبب ضياعاً في مساحة الخزن لكون أن هنالك عدداً كبيراً من سجلات الموجودات في الجدول لا تحمل هذا العدد من المواصفات، وإنما تكتفي باثنين أو ثلاث صفات وتترك باقي حقول الصفات خالية. كذلك عند إضافة موجودات جديدة وتحتاج هذه الموجودات إلى ذكر مواصفات اكثر عندها يجب تعديل بنية الجدول.

NOSQL CouchDB ت

يعد استخدام نموذج وثيقة البيانات هو الأقرب لطبيعة البيانات الموجودة في قاعدة بيانات جرد الموجودات الورقي، إذ يتم خزن كل قائمة ورقية بوثيقة بيانات JSON . يمكن الاستعلام بسهولة عن الموجودات وصفاتها وأماكن تواجدها باستخدام الأدوات واللغات البرمجية التي يوفرها نظام CouchDB وباستخدام أي جزء من الوثيقة في الاستعلام. تم استخدام تطبيق Fauxton عن الموجودات وتفاصيلها كما هو موضح بـ(الشكل4) و(الثكل5) و (الثكل6)، بالإضافة الى ذلك تم تكوين مجموعة من المشاهد حسب الحاجة (مثل تتظيم الوثائق بالاعتماد على وجود نوع محدد من الأغراض أو حسب مواصفات الموجودات أو أسماء المسؤولين ) باعتماد طريقة تقنية المقابلة والتخفيض Map-Reduce كما هو موضح بـ(الثكل7). 
Journal of Education and Science (ISSN 1812-125X), Vol: 29, No: 2, 2020 (82-100)

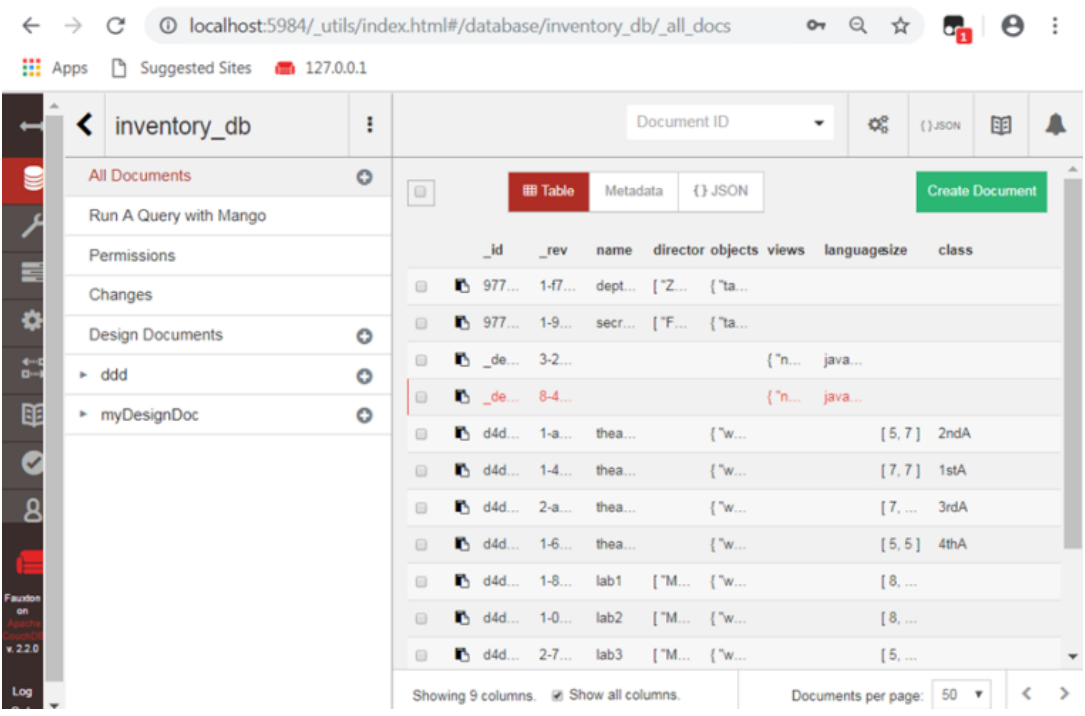

$$
\text { الثكل (4)مكونات قاعدة بيانات الجرد }
$$

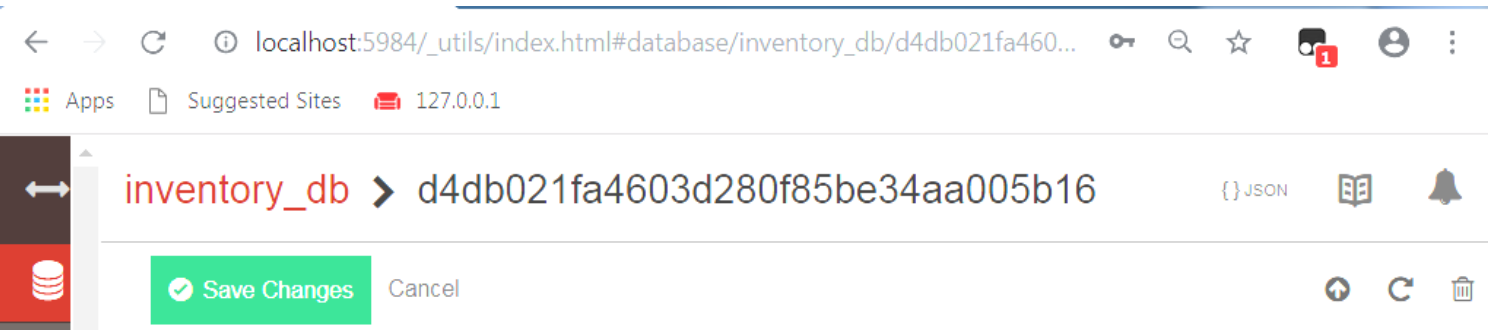

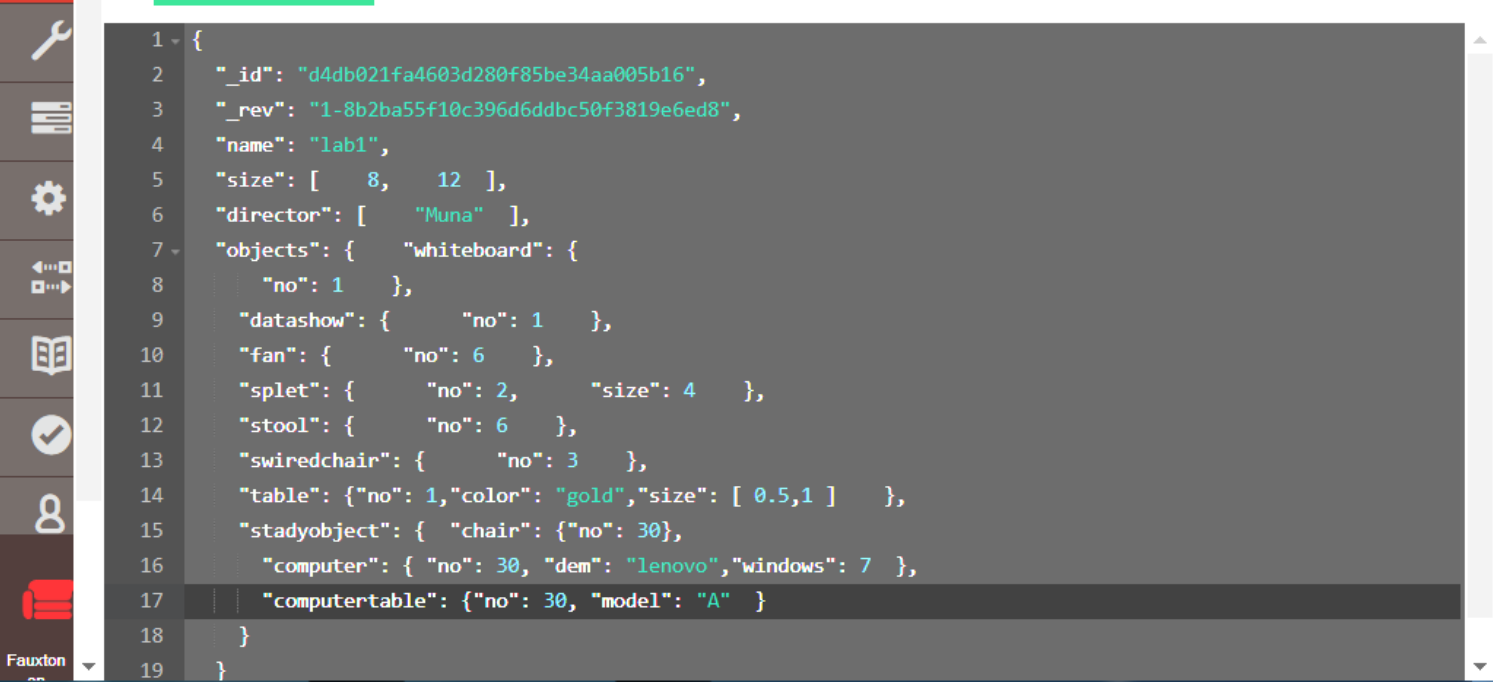

$$
\text { الثكل (5) نموذج وثيقة بيانات لمختبر في قاعدة بيانات الجرد }
$$




\section{Journal of Education and Science (ISSN 1812-125X), Vol: 29, No: 2, 2020 (82-100)}

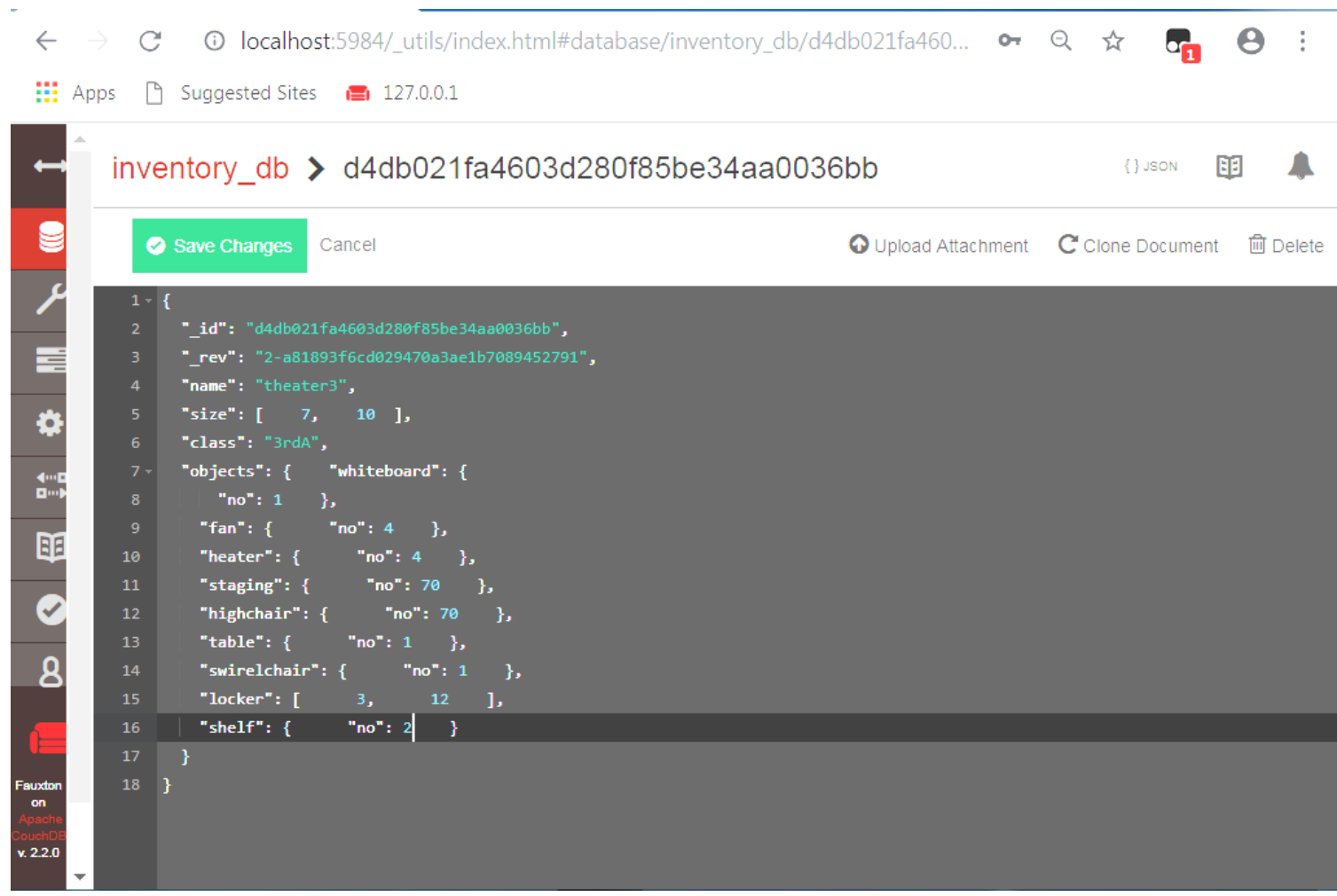

الثكل (6) نموذج وثيقة بيانات لقاعة محاضرات في قاعدة بيانات الجرد

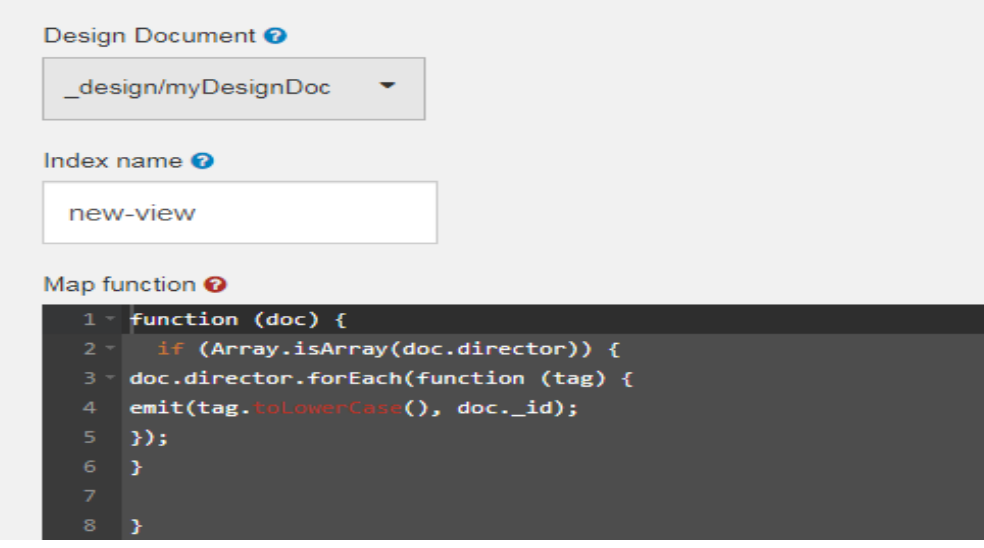

الثكل (7)نموذج تكوين مشهد Map-Reduce في قاعدة بيانات الجرد

Cloudant-Query 3-9 لغة الاستعلام

تم العمل بطريقة Mango Query التي تستخدم لغة Cloudant Query للاستعلام عن بيانات وثائق JSON وتتكون جمل الاستعلام هذه من الاجزاء التالية التي تقابل الأجزاء الموجودة في لغة الاستعلام المهيكلة SQL:

$$
\begin{aligned}
& \text { •selector وهي مجموعة البيانات التي سوف تم استعادتها. } \\
& \text { • • fields الحقول التي سوف يتم استعادتها. } \\
& \text { • • }
\end{aligned}
$$




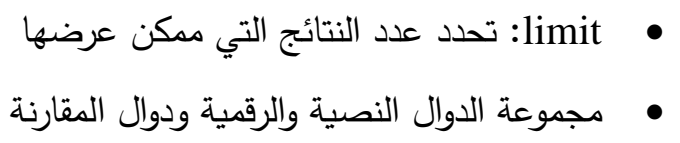

$\{$ \$text, \$gt,\$1t, $\$ \operatorname{in}[],, \ldots\}$

• مجموعة العمليات المنطقية

\{\$and, \$or, \$not $\}$

يوضح (الثكل8) استعلام1 الذي يمثل الاستعلام عن المواقع التي يوجد فيها خزانة locker وهو يقابل تتفيذ الأمر التالي بلغة :SQL

Select name, locker

from inventory

where locker $<>$ null

ويوضح (الثكل9) استعلام2 الذي يمثل الاستعلام عن المواقع التي يديرها أي من [Muna ,Ahmed] وهو يقابل تتفيذ الامر

SQL التالي بلغة

Select name, director

from inventory

where director in ["Muna", "Ahmed"]

$$
\text { ويوضح (الثكل10) استعلام3 الذي يمثل الاستعلام عن المختبرات التي يكون فيها نقص اجهزة حاسوب }
$$

Select name, director, stadyobject

from inventory

where name like ("tab\%") and computer_no< computertable_no

order by name

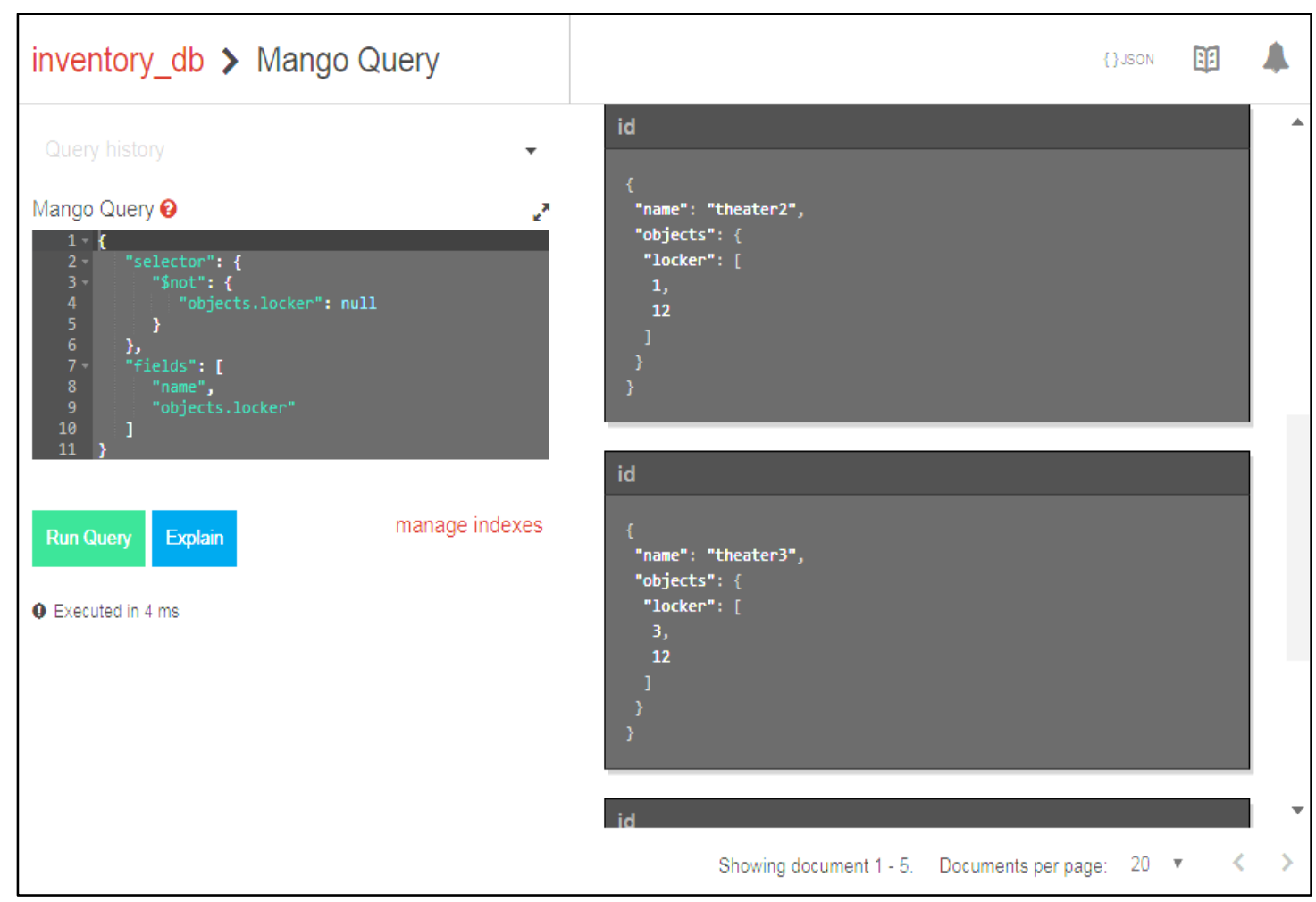

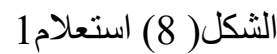




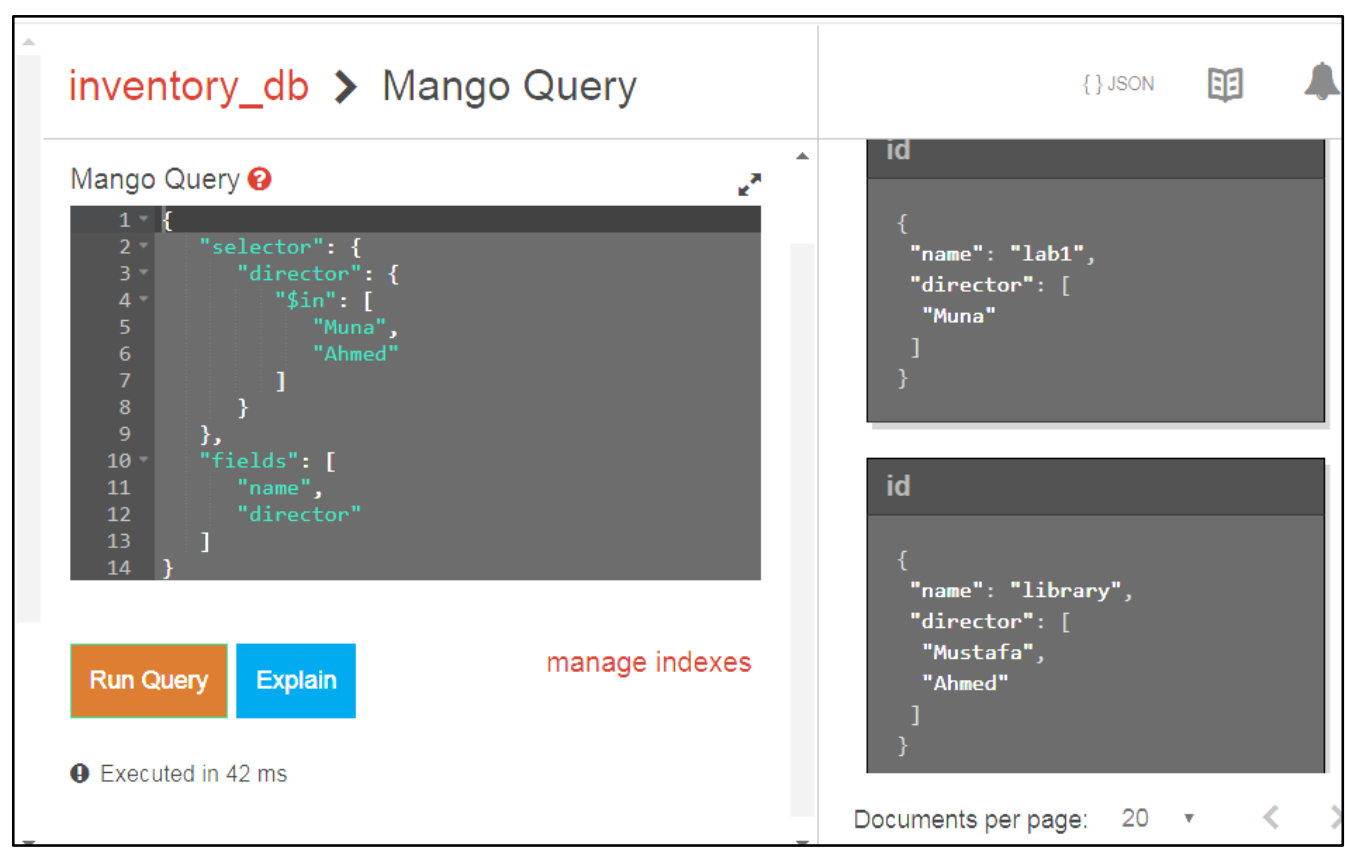

الثكل(9) استعلام2

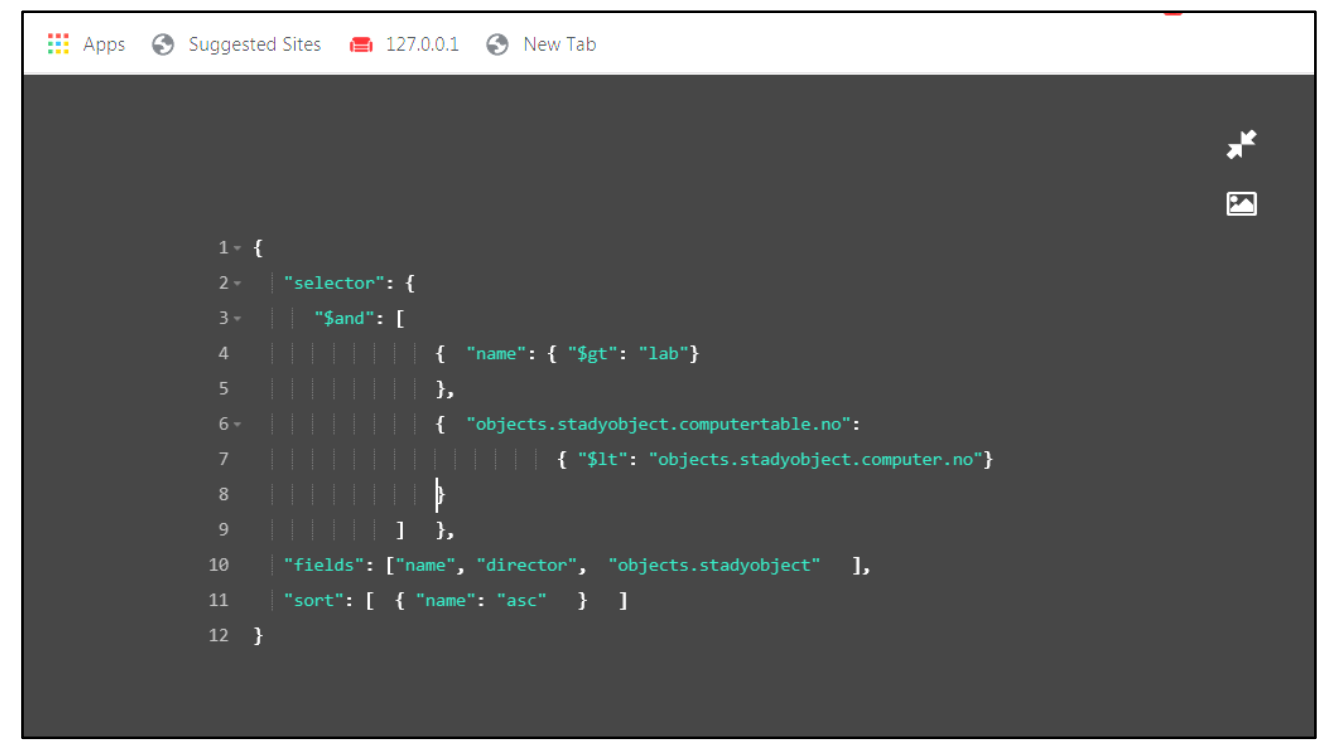

شكل(10) استعلام3

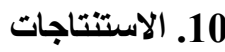

إن كل من قواعد البيانات العلائقية وقواعد بيانات NOSQL لديه مميزات وعيوب وخصائص تميزها ولا توجد قاعدة بيانات أفضل من الأخرى، بل لكل منها استخدامات مختلفة حسب طبيعة النظام المراد تصميمه ويوضح (الجدول 1) اهم الفروقات بين قواعد البيانات العلائقية وقواعد بيانات NOSQL: 
جدول(1) الفروقات بين قواعد البيانات العلائقية وقواعد بيانات NOSQL

\begin{tabular}{|c|c|}
\hline قواعد بيانات NOSQL & قواعد البيانات العلائقية \\
\hline 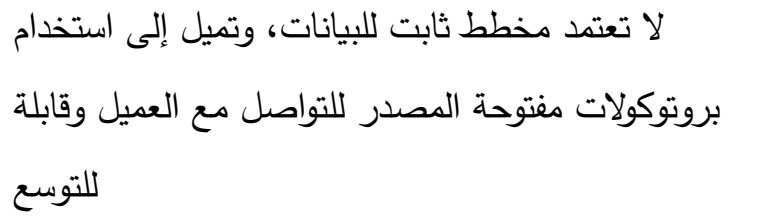 & 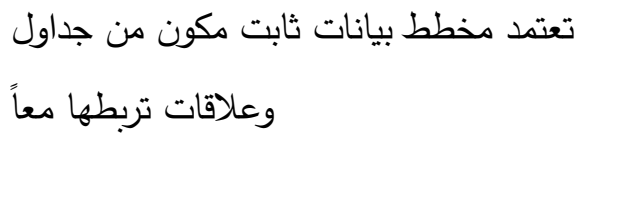 \\
\hline 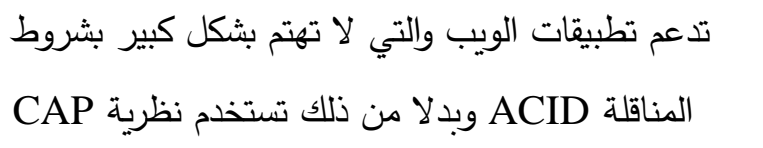 & تدعم معالجة المناقلات وتستخدم شروط المناقلة \\
\hline 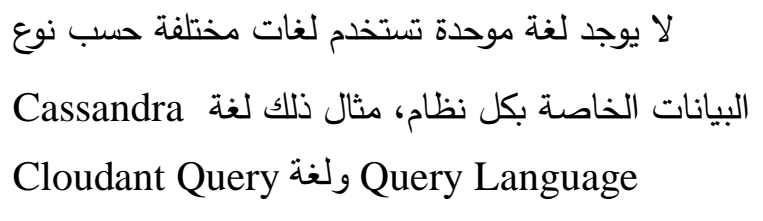 & 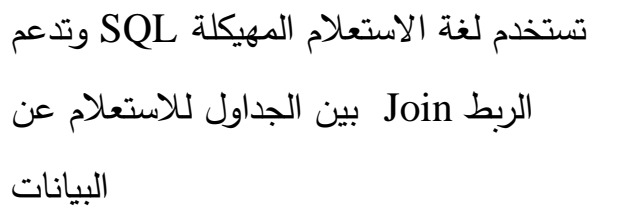 \\
\hline $\begin{array}{l}\text { لا تهتم بقواعد تطبيع البيانات وتتظر لتكرار البيانات على أنه مشكلة يمكن تخطيها } \\
\text { أبنات }\end{array}$ & $\begin{array}{l}\text { Normalization تعتمد مبدأ تطبيع البيانات } \\
\text { للتخلص من تكرار البيانات }\end{array}$ \\
\hline $\begin{array}{l}\text { تدعم المعالجة المتوازية على اكثر من خادم والبيانات } \\
\text { تتبع عدة هياكل منفصلة مختلفة }\end{array}$ & تدعم مبدأ المعالجة المركزية لهياكل البيانات \\
\hline يمكن بسهولة تحجيم هياكل البيانات كونها منفصلة عن & من الصعوبة عمل تحجيم لهياكل البيانات لأنها \\
\hline
\end{tabular}

استخدام قواعد بيانات NOSQL يوفر طريقة جديدة للتعامل مع البيانات شبه المهيكلة وغير المهيكلة إذ تكون البيانات كثيرة التفاصيل وموزعة على مواقع متعددة. تعتبر البيانات الناتجة من جمع المعلومات عن عمليات جرد الموجودات في المؤسسات من مختلف مواقعها مثال جيد عن البيانات شبه المهيكلة والتي من الصعب جداً تنظيمها باستخدام النموذج العلائقي (بثكل جداول مكونة من صفوف واعمدة) وذلك لاختلاف طبيعة الموجودات في كل موقع ومواصفاتها عن المواقع الأخرى، بالإضافة إلى اختلاف طبيعة الموجودات في الموقع الواحد عن بعضها البعض. لذلك فإن تنظيم هذا النوع من البيانات في قواعد NOSQL هو الحل الأمثل وبشكل وثائق بيانات JSON التي تكون ذات طبيعة مرنة.

باستخدام نظام قواعد بيانات CouchDB لبناء قاعدة بيانات جرد الموجودات والاعتماد على نموذج وثيقة البيانات كإدخالات سهل عمليات الاضافة والحذف والتعديل للموجودات في كل موقع مع إمكانية إضافة مواقع جديدة إلى قاعدة بيانات المؤسسة، حتى وإن كانت طبيعة الموجودات المضافة جديدة كلياً لأنها تضاف بشكل وثائق جديدة. الحقول في وثيقة ليس لها شروط ثابتة أو أسماء محددة وتضم انواعاً مختلفة من البيانات، ولا يوجد حد معين لعدد الحقول في الوثيقة ولا 
حد معين لحجم النص أو عناصر المصفوفة، وكذلك يمكن تكرار اسم الحقل أكثر من مرة مع أنواع بيانات مختلفة ويتم التعامل معها عن طريق معرف الوثيقة.

استخدام طريقة العرض بشكل مشاهد ديناميكية تعتمد تقنية المقابلة والتخفيض ولا تؤثر على طبيعة البيانات الاصلية

وتكون متوفرة عند الحاجة، ولا تحتاج الى إعادة تكوين في كل استخدام لها إذ إنها مشاهد مخزنة وتنظم البيانات فيها حسب طريقة العرض المطلوبة.عند التعامل مع قاعدة بيانات الوثيقة ممكن معالجة وطرح استفسارات واستعلامات بالاعتماد على حقول في المجموعة وكذلك يمكن استرجاع جزء من المجموعة بدل المجموعة بالكامل. وأيضا يمكن تكوين فهارس على أجزاء المجموعة. كما يدعم نظام قواعد بيانات CouchDB لغة الاستعلام Cloudant Query وهي لغة قوية تتعامل مع مكونات وثائق JSON لتكون طريقة استرجاع بيانات مشابهة لما تملكه لغة الاستعلام المهيكلة SQL.

المصادر

1. Vera, Harley and Valeria Guimaraes, Wagner Boaventura, CEUR Workshop Proceeding Vol.1478, September 2015, p.129-135.

2. Salehnia, Ali, "Comparisons of Relational Databases with Big Data: a Teaching Approach", South Dakota State University, Brookings, SD 57007.

3. Chandra, Deka G., Future Generation Computer Systems Vol.52, November 2015, p.13-21.

4. Meijer, Robert J. and Jan S. der Veen, IEEE Fifth International Conference on Cloud Computing 2012, June 2012, p.431-438.

5. Varga, Viorica and Katalin Tunde, Acta Polytech. Hungarica Vol.13.2, January 2016, p.229248.

6. Woolf, Hohpe and Bobby Woolf, "Enterprise Integration Patterns". Addison Wesley. 2003. ISBN 0321200683.

7. Fowler, PoEAA., "Patterns of Enterprise Application Architecture". Addison Wesley. 2003. ISBN 0321127420

8. Robert, Daigneau, "Service Design Patterns". Addison-Wesley.2012.ISBN 032154420X

9. Hadjigeorgiou, Christoforos, MSc in High Performance Computing, The University of Edinburgh, 23 August 2013.

10. Mohamed, A. Mohamed and Mohammed O. Ismail, International Journal of Computer and Information Technology Vol.3.3, May 2014, p.598-601.

11. Lynch, Nancy and Seth. Gilbert, Acm Sigact News Vol.33.2, June 2002, p.51-59.

12. Brewer, Eric A., PODC Vol.7, July 2000.

13. Eric., Evans, "Domain-Driven Design". Addison-Wesley. 2004. ISBN 0321125215 
14. Sadalage, Pramod J. and Martin Fowler, "NoSQL Distilled, A Brief Guide to the Emerging World of Polyglot Persistence", 2013 Pearson Education.

15. Gosain, Dishant and Ishita Kathuria, International Journal of Engineering Research \& Technology Vol 1.6, August 2012, p.1-5.

16. Arnicans, Guntis and Girts Karnitis, 7th International Conference on Computational Intelligence, Communication Systems and Networks (CICSyN), 2015.

17. Mason, R. T., "NoSQL databases and data modeling techniques for a document-oriented NoSQL database". Informing Science \& IT Education Conference (InSITE) 2015.

18. Chang, Fay, Jeffrey Dean and S. Ghemawat, ACM Transaction on computer system(TOCS) Vol.26.2, June 2008.

19. Lakshman, Avinash and P. Malik, SIGOPS Oper. Syst. Rev. 44.2 , 2010.

20. Yang, Hung-chih and Ali Dasdan, R. Hsiao, The 2007 ACM SIGMOD international conference on management of data, June 2007, p.1029-1040.

21. Gilbert, Seth and Nancy Lynch, IEEE computer Vol.45.2, January 2012, p.30-36.

22. Drew, Adams, "Oracle Database JSON Developer's Guide", Copyright 2018.

23. Droettboom, Michael, "Understanding JSON Schema", Release 6.0,Space Telescope Science Institute, 2018. 